\title{
Radiological hazards assessment of laterite mining field in Ilorin, North-central Nigeria
}

\author{
M.M. Orosun ${ }^{1 *}$, M.R. Usikalu², K.J. Oyewumi ${ }^{1}$ \\ ${ }^{1}$ Department of Physics, University of Ilorin, Ilorin, Kwara state, Nigeria \\ ${ }^{2}$ Department of Physics, Covenant University, Ota, Ogun State, Nigeria
}

\begin{abstract}
\section{- Original article}

\section{*Corresponding authors: \\ Muyiwa Michael Orosun, Ph.D., E-mail:}

muyiwaorosun@yahoo.com

Revised: November 2019

Accepted: January 2020

Int. J. Radiat. Res., October 2020; 18(4): 895-906

DOI: 10.18869 /acadpub.ijrr.18.4.895

Background: Assessment of activity concentrations of ${ }^{238} \mathrm{U},{ }^{232} \mathrm{Th},{ }^{40} \mathrm{~K}$ and gamma dose rate (DR) was carried out over a laterite mining field in llorinsouth, Nigeria. Materials and Methods: A well calibrated Super-Spec (RS-125) gamma spectrometer was used to measure the activity concentrations of ${ }^{40} \mathrm{~K}$, ${ }^{238} \mathrm{U},{ }^{232} \mathrm{Th}$ and gamma doses rate at $1 \mathrm{~m}$ above the ground level over the laterite mining field. Fifty (50) measurements of the activity concentration of the radionuclides were obtained at about 1 meter above the topsoil. For each point, measurements were taken four (4) times for better accuracy. Results: The overall mean of the activity concentrations of ${ }^{40} \mathrm{~K},{ }^{238} \mathrm{U}$ and ${ }^{232} \mathrm{Th}$ are $81.38,43.89$ and $38.79 \mathrm{Bqkg}^{-1}$ respectively. The mean value for DR was found to be $46.44 \mathrm{nGyhr}^{-1}$. The mean activity concentrations of ${ }^{238} \mathrm{U}$ and ${ }^{232} \mathrm{Th}$ are higher than the recommended limits provided by UNSCEAR. This is a cause for worry as significant enhancement in the concentration of ${ }^{238} \mathrm{U}$ and ${ }^{232} \mathrm{Th}$ will increase the level of the background radiation and possibly render the soil unfit for use in building and construction. The results of most of the radiological impact parameters (RIP) are above the limits provided by UNSCEAR. Conclusion: From the results, it implies that the risk of indoor gamma radiation exposure is high for this lateritic soil. So the lateritic soil from this mine field may not be too suitable for building and construction purposes.
\end{abstract}

Keywords: Radioactivity, background radiation, gamma spectrometry, radiological impact parameters, Ilorin.

\section{INTRODUCTION}

To achieve rapid economic development, third-world countries like Nigeria often resort to mining to exploit natural resources. Consequently, mining is an important economic activity which has the potential of contributing to the development of areas endowed with the resources. It contributes to the revenue of countries; increase the Gross Domestic Product (GDP) and foreign exchange earnings (1). But as desirable and necessary as this is, it became an albatross because of the lack of appropriate policies to guide it in Nigeria. The impact of these mining activities on our environment and health can never be over-emphasized. Recent investigations revealed that mining and smelting activities are the main causes for the increasing pollution of radionuclides and other toxic contaminants (2-4). The enhancement of these radionuclides from the mining, milling and smelting activities can cause potential radiation exposure to members of the public leading to harmful and even lethal effects. The human health effects of the ionizing radiation from these mineral resources hinge on the use of mineral soils from the contaminated sites. These mineral soils, particularly laterites (a reddish clayey soil, rich in aluminum and iron oxides, formed as a result of weathering of igneous rocks in moist temperate climates) are used in building and construction of houses in Nigeria 
$(5,6)$. Although Lateritic soils are sometimes used as an iron ore and a source of nickel, they are widely used as construction materials for houses, roads, dam embankment, landfills and flexible pavement foundations, etc. Because blocks made from these soils (laterites) are naturally obtainable (available), cheap, energy efficient to produce and have good geotechnical properties, it becomes a handy material for construction that even the underprivileged and low class or low income earners can afford (7). Considering the radiological health implications linked with the exposure to indoor ionizing radiation, regulatory bodies such as United State Environmental Protection Agency (USEPA), the World Health Organization (WHO), and International Commission on Radiological Protection (ICRP) etc. have implemented stringent measures intended to reduce such exposures. Consequently, the call for the measurement and assessment of natural radionuclides in soils used for building and construction purposes (particularly soils around mining, milling and smelting industries) worldwide becomes obligatory $(5,8,9)$.

In Nigeria, the levels of ${ }^{238} \mathrm{U},{ }^{234} \mathrm{Th}$ and their respective progenies together with the non-series ${ }^{40} \mathrm{~K}$ have been studied in different mineral soils from many parts of the country $(10-19)$. But there is no data on the activity concentration of natural radionuclides ${ }^{238} \mathrm{U}$, ${ }^{234} \mathrm{Th}$ and ${ }^{40} \mathrm{~K}$ ) in Laterites in this part of the country, despites the level of the mining activities. Also, data from University of Ilorin Teaching Hospital (UITH) shows that 74 different cancers of 2,246 (891 male and 1355 female) cancer patients within the age of 1-105 were recorded at the University of Ilorin Teaching Hospital (UITH) cancer registry between the period of 2007 and 2016 [20]. Hence the aim of this research work is to assess the activity concentrations of ${ }^{238} \mathrm{U},{ }^{232} \mathrm{Th},{ }^{40} \mathrm{~K}$ and gamma dose rate (DR) over the Laterite mine field in Ilorin, North central Nigeria using a well calibrated Super-Spec (RS-125) gamma and use the results to estimate the radiation absorbed dose rates, annual effective dose, external and internal radiation hazard indices and other radiological hazard parameters. The results of this research will serve as a firsthand information that can be used to assess the radiological health implications on the general populace due to the use of laterites from the mining sites in Ilorin-south, Kwara, North central Nigeria. This will help the Government to make policies that will help to regulate the mining activities and keep the standard of living of the people as high as possible.

\section{Study area}

The study area is along Ajasse-Ipo road in Ilorin-south LGA in Kwara state, Nigeria. It is

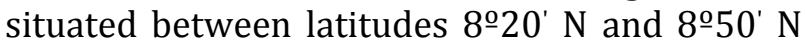
and Longitudes $4^{\circ} 25^{\prime} \mathrm{E}$ and $4^{\circ}-65^{\prime} \mathrm{E}$ (figure $1 \mathrm{a}$ and $b$ ). For the geology of the study area, a large part of Ilorin town is underlain by basement complex rock. The soils are formed from basement complex rocks (metamorphic and igneous rocks) which is about 95\%. The metamorphic rocks consist of biotite gnesiss, banded gnesiss, quartzite augitegnesiss and granitic gnesiss. The intrusive rock comprises of pegmatite and vein quartz $(21,22,23)$. The assortment of basement complex rocks brings about large number of ferruginous groups of soils. Therefore, lateritic soil type (generally deep red in colour with high clay content) is the major type of soil in Ilorin. Detail geology of Ilorin can be found in $(22,23,24)$.

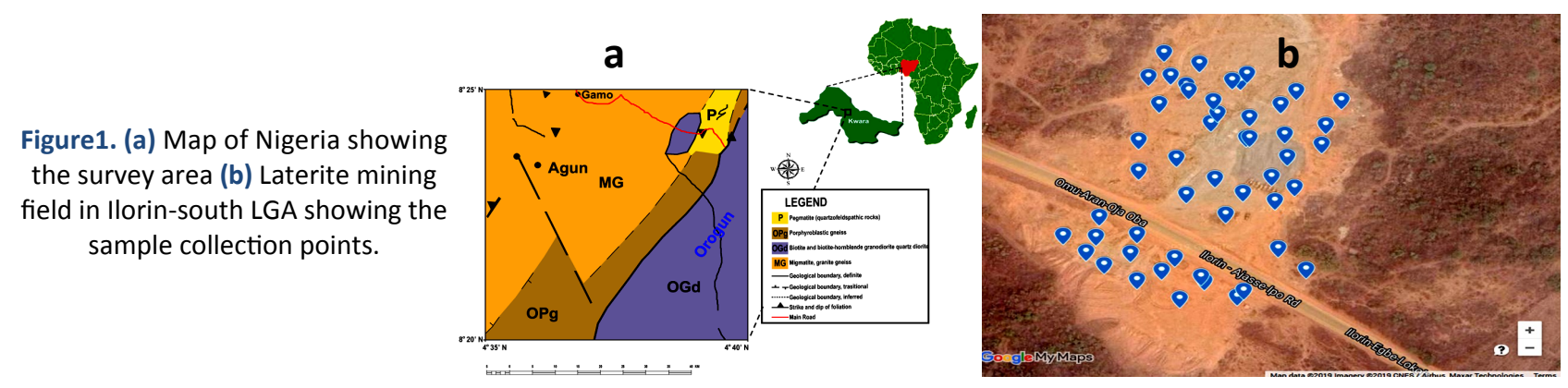




\section{MATERIALS AND METHODS}

\section{Field Survey}

For the in situ measurements of activity concentrations of ${ }^{40} \mathrm{~K},{ }^{232} \mathrm{Th},{ }^{238} \mathrm{U}$ and the radiation dose exposures, Super SPEC RS-125 spectrometer with large $2.0 \times 2.0 \mathrm{NaI}$ crystal was used. The measurement of the activity concentration of the radionuclides was carried out at about 1 metre above the topsoil $(19,25)$. The RS-125 is a transportable handheld radiation detector with high accuracy and likely error of about 5\%. It presents superior integrated design with big detector, good sensitivity and easy to use. The model RS-125 super-spec is manufactured by Canadian Geophysical Institute, Canada. It comes with a large data storage which allows one to take multiple readings with ease. The RS-125 spectrometer was calibrated in accordance with Canadian Geophysical Institute i.e., the instrument was calibrated on $1 \times 1 \mathrm{~m}$ test pads, which employs $5 \mathrm{~min}$ spectra accumulation on potassium, uranium and thorium pads and $10 \mathrm{~min}$ accumulation on the Background pad. It makes use of sodiumiodide (NaI) crystal doped with thallium [Tl] as activator. The energy range of the instrument, is from 30 to $3000 \mathrm{keV}$, which is enough to detect most of the radiation giving off from the terrestrial sources (i.e. ${ }^{214} \mathrm{Bi}$ (609.31 and $1764.49 \mathrm{keV}$ ) gamma rays to determine ${ }^{238 \mathrm{U},}{ }^{212} \mathrm{~Pb}(238.63 \mathrm{keV}),{ }^{208} \mathrm{Tl}(583.19 \mathrm{keV})$ and ${ }^{228} \mathrm{Ac}(911.21 \mathrm{keV})$ gamma rays to determine ${ }^{232} \mathrm{Th}$ and the photopeaks of ${ }^{40} \mathrm{~K}$ which occours in the background spectrum at $1460.83 \mathrm{keV}$ ). The total count of $120 \mathrm{~s}$ per assay was employed for best accuracy as stated in Radiation Solutions Inc (Radiation solution Inc, 2015; Adgunodo et al., $645^{2}$ ). The assay mode of the instrument gives the activity concentration of ${ }^{40} \mathrm{~K}$ in percentage (\%), ${ }^{238} \mathrm{U}$ and ${ }^{232} \mathrm{Th}$ in part per million (ppm). The data was converted to the conventional unit $B q \mathrm{~kg}^{-1}$ using conversion factors given by $(26,27)$.

In this work, four (4) readings were recorded at each data point at the interval of 120 seconds. 50 sample points were recorded to cover the area of the mining field. The field was divided into grids of approximately equal size (i.e. 50 semi-rectangular boxes) with each box Int. J. Radiat. Res., Vol. 18 No. 4, October 2020 representing a data collection point. At each of these samples location (point), the coordinate and elevation were determined using a global positioning system (GPSMAP78). More details about the instrument can be found in earlier works where this same Super SPEC RS-125 spectrometer was used $(19,25,28,29)$.

\section{Estimation of the radiological impact parameters (RIP) Absorbed dose rate}

Estimating the absorbed dose rate is usually the initial step for evaluating the health risk. The biological effects of ionizing radiation are unswervingly associated with the absorbed dose rate [30]. The outdoor absorbed dose rate at 1 meter height above the ground level (it is assumed that the naturally occurring radionuclides will have a uniform distribution at this height) is calculated using equation (1) $(16,25$, $31,32,33)$.

$\mathrm{D}_{\text {outdoor }}\left(\mathrm{nGy} \mathrm{h}^{-1}\right)=0.462_{\mathrm{u}}+0.604 \mathrm{C}_{\mathrm{Th}}+0.041 \mathrm{C}_{\mathrm{K}}$

But fortunately, this outdoor dose rate was measured in situ using the RS-125 Gamma Spec.

The laterite from study area as emphasized earlier, is used predominantly for building purposes. Consequently, the indoor radiation dose rate in a characteristic building of typical room $4 \times 5 \times 2.8 \mathrm{~m}$ size having wall thickness of about $20 \mathrm{~cm}$ and density of the structure 2350 $\mathrm{kgm}^{-3}$ was calculated using the equation provided by (31) and (34) for building materials as used by numerous researchers $(16,31,34,35,36,37)$ :

$D_{\text {intdoor }}\left(\right.$ nGy h$\left.^{-1}\right)=0.92 C_{u}+1.1 C_{T h}+0.08 C_{K}$

$C_{u}, C_{T h}$ and $C_{K}$ are the radioactivity concentration in $\mathrm{Bq} \mathrm{kg}^{-1}$ for ${ }^{238} \mathrm{U},{ }^{232} \mathrm{Th}$ and ${ }^{40} \mathrm{~K}$ respectively.

\section{Annual effective dose (AED)}

The effective dose which is the tissue-weighted aggregate of the equivalent doses in all specified tissues and organs of the body. It corresponds to the stochastic health risks to the whole body. The annual effective dose received indoor and outdoor by a member 
of the public was calculated from dose rates given in equations (1) and (2) by using dose conversion factor of $0.7 \mathrm{SvGy}^{-1}$ and occupancy factor for outdoor and indoor as 0.2 and 0.8 respectively $(16,31)$.

$\mathrm{AED}_{\text {outdoor }}\left(m S v y^{-1}\right)=\mathrm{D}_{\text {outdoor }}\left(n G y h^{-1}\right) \times 8760 h \times$ $0.7\left(S v G y^{-1}\right) \times 0.2 \times 10^{-6}$

$\operatorname{AED}_{\text {intdoor }}\left(m S v y^{-1}\right)=D_{\text {indoor }}\left(n G y h^{-1}\right) \times 8760 h \times$ $0.7\left(S v G y^{-1}\right) \times 0.8 \times 10^{-6}$

\section{Radium equivalent activity index ( $\left.R a_{e q}\right)$}

The radium equivalent $\left(R a_{e q}\right)$ activity which is a weighted sum of activities of ${ }^{238} \mathrm{U},{ }^{232} \mathrm{Th}$ and ${ }^{40} \mathrm{~K}$ based on the assumption that $5 \mathrm{~Bq} \mathrm{~kg}^{-1}$ of ${ }^{238} \mathrm{U}$, $0.7 \mathrm{Bqkg}^{-1}$ of ${ }^{232} \mathrm{Th}^{2}$ and $57 \mathrm{Bqkg}^{-1}$ of ${ }^{40} \mathrm{~K}$ produce the same radiation dose rates. This permit a solitary index or figure to portray the gamma output due to different combination of $238 \mathrm{U}$, ${ }^{232} \mathrm{Th}$ and ${ }^{40} \mathrm{~K}$ in a material. This radiation hazard index estimates the appropriateness of the laterite soil to be used as a building material. It was calculated using equation (5) (38):

$\mathrm{Ra}_{\mathrm{eq}}=\mathrm{C}_{\mathrm{u}}+1.43 \mathrm{C}_{\mathrm{Th}}+0.077 \mathrm{C}_{\mathrm{K}}$

$C_{U}, C_{T h}$ and $C_{K}$ are as defined in equation (1) and (2) above. The recommended average value for $R a_{e q}$ is $370 \mathrm{~Bq} \mathrm{~kg}^{-1}$.

\section{Radiation hazard indices}

The external radiation hazard $\left(H_{e x t}\right)$ and the internal radiation hazard $\left(H_{\text {int }}\right)$ were calculated using equation 6 and 7 .

$$
\begin{aligned}
& H_{\text {ext }}=\left(\frac{C_{U}}{370}\right)+\left(\frac{C_{T h}}{259}\right)+\left(\frac{C_{K}}{4810}\right) \\
& H_{\text {int }}=\left(\frac{C_{U}}{185}\right)+\left(\frac{C_{T h}}{259}\right)+\left(\frac{C_{K}}{4810}\right)
\end{aligned}
$$

$H_{\text {int }}$ and $H_{\text {ext }}$ ought to be less than 1 for the radiation hazard to be insignificant. Natural radionuclides in soil produce an external field to which all humans are exposed. $H_{\text {ext }}$ equal to unity translates to the upper limit of radium equivalent dose $\left(370 \mathrm{~Bq} \mathrm{~kg}^{-1}\right)(31,38)$.

\section{Excess lifetime cancer risk (ELCR)}

The Excess Lifetime Cancer Risk (ELCR) was calculated using the following equation:

$E L C R=A E D_{\text {indoor }} \times D L \times R F$

$A E D_{\text {indoor }}$ is the indoor annual equivalent dose should the laterite be used for building construction, $D L$ is the average duration of life (estimated to 70 years) and $R F$ is the risk factor $\left(S v^{-1}\right)$, i.e. fatal cancer risk per Sievert $(31,39)$.

\section{Annual gonadal equivalent dose (AGED)}

There are some organs that are considered of interest by UNSCEAR because of their sensitivity to radiation. These organs include; the gonads, the bone marrow and the bone surface cells [31]. An increase in $A G E D$ has been known to result in leukemia which is very fatal. This hazard parameter for the residents using the laterite for building was evaluated using equation $(9)(31,38)$ :

$A G E D\left(\mu S v y^{-1}\right) \mathrm{C}=3.09 C_{U}+4.18 C_{T h}+0.314 C_{K}$

$C_{U}, C_{T h}$, and $C_{K}$ maintain their usual meanings.

\section{Representative Level Index (RLI)}

This hazard parameter estimates the gamma radiation hazard associated with the natural radionuclide in the samples under examination. The $R L I$ was estimated using equation $10(31,38)$ :

$R L I=\frac{C_{u}}{150}+\frac{C_{T h}}{100}+\frac{C_{k}}{1500} \leq 1$

$C_{U}, C_{T h}$ and $C_{K}$ maintain their usual meanings.

$R L I$ values of 5 corresponds to an $A E D$ of less than or equal to $1 \mathrm{mSv}$, while $R L I=0.5$ corresponds to $A E D$ less or equal to $0.3 \mathrm{mSvy}^{-1}$. Thus, RLI serves as a screening tool for identifying building materials that may possibly be of concern to be used as construction materials $(31,38)$. 


\section{RESULTS}

The result and statistical analysis (Min, Max, Median, Mean, STDEV, SKE and KURT) of the measured activity concentrations of ${ }^{40} \mathrm{~K},{ }^{238} \mathrm{U}$ and ${ }^{232} \mathrm{Th}$, the elevations and the dose rate for the 94 sample locations is presented in table 1 . The measured values for all the parameters (i.e. ${ }^{238} \mathrm{U}$, ${ }^{232} \mathrm{Th},{ }^{40} \mathrm{~K}$ and $\mathrm{DR}$ ) were moderately skewed (the distribution is approximately or moderately symmetric) since most of the measure of the asymmetry of their probability distribution about their means is in the range of -2 and +2 [40]. As expected, the mean activity concentration of ${ }^{40} \mathrm{~K}$ is higher than the ${ }^{238} \mathrm{U}$ and ${ }^{232} \mathrm{Th}$ mean activities. ${ }^{40} \mathrm{~K}$ has highest activity concentration of $187.80 \pm 2.44 \mathrm{~Bq} \mathrm{~kg}^{-1}$ and lowest value of $31.30 \pm 1.00 \mathrm{~Bq} \mathrm{~kg}^{-1}$. The highest and lowest activity concentrations of ${ }^{238} \mathrm{U}$ and ${ }^{232} \mathrm{Th}$ were found to be ${ }^{1} 9.78 / 6.66,{ }^{0} 9 .{ }^{11} / 6.4^{3}$ $\mathrm{Bqkg}^{-1}$ and $21.00 \pm 0.82,16.65 \pm 2.66 \mathrm{Bqkg}^{-1}$ respectively. The overall mean of the activity concentrations of the measured radionuclides was calculated and found to be $81.38,43.89$ and $38.79 \mathrm{Bqkg}^{-1}$ for ${ }^{40} \mathrm{~K},{ }^{238} \mathrm{U}$ and ${ }^{232} \mathrm{Th}$ respectively. The measured values and estimated mean value for ${ }^{40} \mathrm{~K}$ were lower than the global average of $420.00 \mathrm{~Bq} \mathrm{~kg}^{-1}$ for normal background radiation levels given by UNSCEAR (figure 2). Surprisingly, the mean activity concentrations of $238 \mathrm{U}$ and ${ }^{232} \mathrm{Th}$ are higher than their corresponding global average of $32.00 \mathrm{Bqkg}^{-1}$ and $30.00 \mathrm{Bqkg}^{-1}$ respectively provided by UNSCEAR. This is a cause for worry as significant enhancement in the concentration of ${ }^{238} \mathrm{U}$ and ${ }^{232} \mathrm{Th}$ will increase the level of the background radiation and possibly render the soil unfit for use in building and construction purposes. The values for the measured outdoor dose rate (DR) ranges between $70.80 \pm 6.23$ and $30.10 \pm 2.03 n G y h^{-1}$ with an average value of $46.44 n G y h^{-1}$. This mean value for the outdoor dose is lower than 59 $n G y h^{-1}$ provided by UNSCEAR.

Radiological impact parameters (RIP) were estimated to evaluate the radiological risks that are associated with the lateritic soil from Ilorin-south LGA. The estimated hazards parameters are presented in table 2. The outdoor absorbed dose rate $\left(D_{\text {out }}\right)$ at 1 meter height above the level ground was estimated using equation 1 . The resulting values of the outdoor absorbed dose rate were used to estimate outdoor annual effective dose $\left(A E D_{\text {outdoor }}\right)$ using equation 3 . The estimated mean values for the $D_{\text {out }}$ and $A E D_{\text {outdoor }}$ are 47.04 $n G y h^{-1}$ and $0.06 \mathrm{mSvy}^{-1}$ respectively. These values are lower than $59.00 n^{n} G h^{-1}$ and 0.07 $m S v y^{-1}$ provided by UNSCEAR. The indoor gamma dose $\left(D_{i n}\right)$ incurred by the general public as a result of the radionuclides concentration in the lateritic soil from the mine field ranges between 135.68 and $58.58 n G y h^{-1}$ with an average value of $89.56 n G y h^{-1}$. The $D_{\text {in }}$ was used to estimate indoor annual effective dose $\left(A E D_{\text {indoor }}\right)$ using equation 4 . The resulting highest, lowest and mean values of $A E D_{\text {indoor }}$ are $0.67,0.29$ and $0.44 \mathrm{mSvy}^{-1}$ respectively. The estimated mean values of $D_{\text {in }}$ and $A E D_{\text {indoor }}$ are above the recommended limits of 84.00 $n G y h^{-1}$ and $0.41 \mathrm{mSvy}^{-1}$ respectively provided by UNSCEAR $(9,16,17,19,31)$. This follows that the risk of indoor gamma radiation exposure is high for this lateritic soil and the general public are not safe from overexposure to indoor ionizing radiation.

Figure 2. Isopotassium map of the laterite mining field.
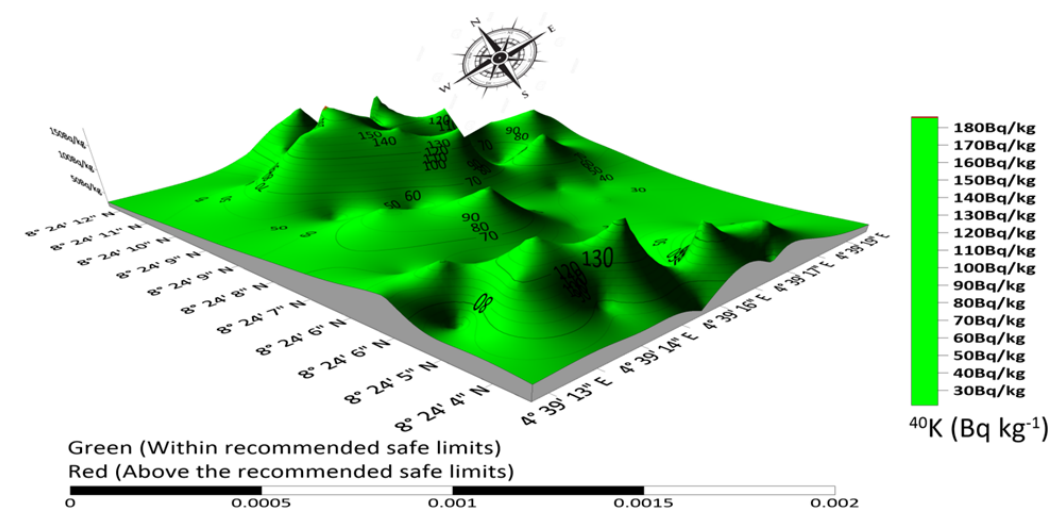
Table 1. Measured activity concentrations of ${ }^{40} \mathrm{~K},{ }^{238} \mathrm{U},{ }^{232} \mathrm{Th}$ and the absorbed dose rates from llorin-south LGA.

\begin{tabular}{|c|c|c|c|c|c|c|c|}
\hline SAMPLE Code & Latitude ${ }^{\circ} \mathrm{N}$ & Longitude ${ }^{\circ} E$ & Elevatn $(m)$ & $\mathrm{DR}\left(n G y h^{-1}\right)$ & ${ }^{40} \mathrm{~K}\left(\mathrm{Bqkg}{ }^{-1}\right)$ & ${ }^{238} \cup\left(B q k g^{-1}\right)$ & ${ }^{232} \mathrm{Th}\left(B q k g^{-1}\right)$ \\
\hline ISS1 & 8.401632 & 4.653531 & 341 & $44.00 \pm 1.13$ & $93.90 \pm 2.00$ & $23.47 \pm 3.66$ & $49.94 \pm 2.22$ \\
\hline ISS2 & 8.401484 & 4.653681 & 342 & $35.10 \pm 2.52$ & $31.30 \pm 2.00$ & $27.17 \pm 2.20$ & $37.76 \pm 4.61$ \\
\hline ISS3 & 8.401367 & 4.653799 & 344 & $48.20 \pm 1.91$ & $125.20 \pm 3.60$ & $30.88 \pm 2.11$ & $49.13 \pm 2.02$ \\
\hline ISS4 & 8.401229 & 4.654014 & 341 & $48.00 \pm 3.07$ & $62.60 \pm 1.03$ & $35.82 \pm 1.00$ & $49.53 \pm 1.98$ \\
\hline ISS5 & 8.401059 & 4.654293 & 343 & $35.70 \pm 3.10$ & $31.30 \pm 6.30$ & $38.29 \pm 1.63$ & $29.23 \pm 4.05$ \\
\hline ISS6 & 8.401080 & 4.654668 & 342 & $31.20 \pm 2.22$ & $62.60 \pm 2.63$ & $21.00 \pm 0.82$ & $33.70 \pm 2.22$ \\
\hline ISS7 & 8.401218 & 4.654454 & 342 & $65.90 \pm 6.51$ & $156.50 \pm 3.03$ & $64.22 \pm 2.01$ & $50.34 \pm 5.44$ \\
\hline ISS8 & 8.401314 & 4.654175 & 342 & $62.00 \pm 2.93$ & $187.80 \pm 2.44$ & $62.99 \pm 2.00$ & $41.82 \pm 2.11$ \\
\hline ISS9 & 8.401473 & 4.653971 & 341 & $48.30 \pm 2.05$ & $156.50 \pm 2.61$ & $59.28 \pm 1.05$ & $24.36 \pm 2.02$ \\
\hline ISS10 & 8.401622 & 4.653746 & 339 & $39.00 \pm 1.32$ & $125.20 \pm 2.60$ & $33.35 \pm 2.11$ & $30.86 \pm 1.08$ \\
\hline ISS11 & 8.401802 & 4.653767 & 339 & $31.20 \pm 1.07$ & $62.60 \pm 2.20$ & $24.70 \pm 2.04$ & $29.64 \pm 2.33$ \\
\hline ISS12 & 8.401643 & 4.654014 & 341 & $44.20 \pm 2.44$ & $31.30 \pm 2.20$ & $30.88 \pm 2.82$ & $48.31 \pm 2.04$ \\
\hline ISS13 & 8.401431 & 4.654260 & 342 & $52.10 \pm 3.20$ & $31.30 \pm 1.60$ & $44.46 \pm 1.08$ & $50.34 \pm 2.20$ \\
\hline ISS14 & 8.401261 & 4.654432 & 340 & $52.10 \pm 4.32$ & $31.30 \pm 4.30$ & $33.35 \pm 0.98$ & $59.28 \pm 2.20$ \\
\hline ISS15 & 8.401133 & 4.654711 & 341 & $30.10 \pm 2.03$ & $125.20 \pm 2.63$ & $27.17 \pm 1.02$ & $21.52 \pm 4.12$ \\
\hline ISS16 & 8.401324 & 4.655119 & 339 & $30.20 \pm 1.83$ & $31.30 \pm 1.85$ & $35.82 \pm 1.44$ & $21.52 \pm 2.01$ \\
\hline ISS17 & 8.401515 & 4.654936 & 340 & $34.80 \pm 2.22$ & $31.30 \pm 2.13$ & $45.70 \pm 2.04$ & $21.11 \pm 1.02$ \\
\hline ISS18 & 8.401823 & 4.654593 & 341 & $32.50 \pm 1.45$ & $31.30 \pm 5.65$ & $46.93 \pm 1.80$ & $16.65 \pm 2.66$ \\
\hline ISS19 & 8.402004 & 4.654336 & 341 & $59.20 \pm 2.84$ & $125.20 \pm 1.06$ & $49.40 \pm 2.21$ & $53.19 \pm 2.81$ \\
\hline ISS20 & 8.402216 & 4.654024 & 341 & $55.40 \pm 5.47$ & $93.90 \pm 2.20$ & $49.40 \pm 2.28$ & $49.13 \pm 2.23$ \\
\hline ISS21 & 8.402492 & 4.654024 & 339 & $55.00 \pm 3.74$ & $31.30 \pm 4.61$ & $59.28 \pm 1.02$ & $44.66 \pm 2.67$ \\
\hline ISS22 & 8.402333 & 4.654271 & 340 & $32.10 \pm 2.13$ & $31.30 \pm 1.40$ & $30.88 \pm 1.05$ & $29.23 \pm 1.82$ \\
\hline ISS23 & 8.402147 & 4.654523 & 341 & $50.00 \pm 4.10$ & $31.30 \pm 2.60$ & $71.63 \pm 2.20$ & $25.98 \pm 2.12$ \\
\hline ISS24 & 8.402020 & 4.654706 & 342 & $33.10 \pm 2.11$ & $31.30 \pm 1.20$ & $25.94 \pm 1.02$ & $33.70 \pm 2.01$ \\
\hline ISS25 & 8.401945 & 4.654915 & 341 & $70.80 \pm 6.23$ & $31.30 \pm 1.60$ & $75.34 \pm 2.22$ & $58.06 \pm 2.51$ \\
\hline ISS26 & 8.402067 & 4.655044 & 342 & $46.10 \pm 2.02$ & $31.30 \pm 2.61$ & $59.28 \pm 2.08$ & $30.04 \pm 2.20$ \\
\hline ISS27 & 8.402163 & 4.654894 & 341 & $37.20 \pm 1.21$ & $31.30 \pm 2.63$ & $55.58 \pm 2.00$ & $20.71 \pm 1.20$ \\
\hline ISS28 & 8.402513 & 4.654725 & 341 & $40.10 \pm 2.75$ & $156.50 \pm 3.11$ & $44.46 \pm 1.08$ & $22.33 \pm 0.95$ \\
\hline ISS29 & 8.402651 & 4.654494 & 342 & $49.50 \pm 3.12$ & $156.50 \pm 2.60$ & $32.11 \pm 0.75$ & $46.69 \pm 2.33$ \\
\hline ISS30 & 8.402821 & 4.654161 & 342 & $40.20 \pm 2.19$ & $156.50 \pm 1.02$ & $32.11 \pm 2.60$ & $31.67 \pm 2.21$ \\
\hline ISS31 & 8.403076 & 4.654231 & 341 & $65.90 \pm 2.02$ & $93.90 \pm 2.60$ & $49.40 \pm 2.07$ & $65.77 \pm 2.09$ \\
\hline ISS32 & 8.402991 & 4.654338 & 339 & $55.10 \pm 2.32$ & $31.30 \pm 2.54$ & $59.28 \pm 2.06$ & $44.66 \pm 2.05$ \\
\hline ISS33 & 8.402736 & 4.654537 & 341 & $48.90 \pm 2.05$ & $93.90 \pm 2.28$ & $35.82 \pm 2.22$ & $49.94 \pm 2.22$ \\
\hline ISS34 & 8.402513 & 4.654746 & 342 & $36.30 \pm 1.55$ & $31.30 \pm 1.82$ & $27.17 \pm 1.20$ & $37.76 \pm 2.07$ \\
\hline ISS35 & 8.402343 & 4.654993 & 344 & $58.60 \pm 4.32$ & $125.20 \pm 4.63$ & $30.88 \pm 2.33$ & $64.96 \pm 2.02$ \\
\hline ISS36 & 8.402455 & 4.655218 & 341 & $49.00 \pm 2.12$ & $62.60 \pm 2.60$ & $35.82 \pm 1.60$ & $49.53 \pm 2.11$ \\
\hline ISS37 & 8.402545 & 4.654977 & 343 & $36.30 \pm 2.08$ & $31.30 \pm 2.54$ & $38.29 \pm 4.63$ & $29.23 \pm 1.88$ \\
\hline ISS38 & 8.402699 & 4.654746 & 342 & $44.30 \pm 2.37$ & $62.60 \pm 2.21$ & $46.93 \pm 3.32$ & $33.70 \pm 4.22$ \\
\hline ISS39 & 8.402847 & 4.654515 & 342 & $46.00 \pm 1.34$ & $156.50 \pm 2.26$ & $51.87 \pm 2.11$ & $25.98 \pm 5.22$ \\
\hline ISS40 & 8.402943 & 4.654354 & 342 & $58.90 \pm 2.87$ & $187.80 \pm 4.10$ & $58.05 \pm 2.00$ & $41.82 \pm 2.11$ \\
\hline ISS41 & 8.403065 & 4.654086 & 343 & $36.30 \pm 1.44$ & $31.30 \pm 1.33$ & $38.29 \pm 1.00$ & $29.23 \pm 2.02$ \\
\hline ISS42 & 8.403283 & 4.654193 & 342 & $49.30 \pm 2.21$ & $62.60 \pm 2.60$ & $59.28 \pm 4.20$ & $33.70 \pm 3.22$ \\
\hline ISS43 & 8.403187 & 4.654419 & 342 & $51.10 \pm 2.09$ & $156.50 \pm 2.24$ & $62.99 \pm 2.44$ & $25.98 \pm 3.04$ \\
\hline ISS44 & 8.403028 & 4.654687 & 342 & $61.10 \pm 4.02$ & $187.80 \pm 2.54$ & $62.99 \pm 2.42$ & $41.82 \pm 2.64$ \\
\hline ISS45 & 8.402816 & 4.654950 & 341 & $51.80 \pm 5.92$ & $156.50 \pm 5.40$ & $69.16 \pm 2.05$ & $24.36 \pm 4.56$ \\
\hline ISS46 & 8.402630 & 4.655250 & 339 & $38.80 \pm 2.21$ & $125.20 \pm 6.20$ & $33.35 \pm 1.08$ & $30.86 \pm 3.04$ \\
\hline ISS47 & 8.402853 & 4.655347 & 339 & $31.10 \pm 2.35$ & $62.60 \pm 2.60$ & $24.70 \pm 1.03$ & $29.64 \pm 2.62$ \\
\hline ISS48 & 8.402938 & 4.655052 & 341 & $44.10 \pm 2.73$ & $31.30 \pm 1.42$ & $30.88 \pm 2.60$ & $48.31 \pm 2.88$ \\
\hline ISS49 & 8.403092 & 4.654735 & 342 & $60.50 \pm 3.75$ & $31.30 \pm 1.50$ & $44.46 \pm 2.88$ & $64.15 \pm 2.21$ \\
\hline ISS50 & 8.403032 & 4.654638 & 340 & $65.50 \pm 2.01$ & $31.30 \pm 1.00$ & $64.22 \pm 4.23$ & $57.65 \pm 3.01$ \\
\hline Min & & & 339.00 & $30.10 \pm 2.03$ & $31.30 \pm 1.00$ & $21.00 \pm 0.82$ & $16.65 \pm 2.66$ \\
\hline Max & & & 344.00 & $70.80 \pm 6.23$ & $187.80 \pm 2.44$ & $75.34 \pm 2.22$ & $65.77 \pm 2.09$ \\
\hline Mean & & & 341.22 & 46.44 & 81.38 & 43.89 & 38.79 \\
\hline Median & & & 47.05 & 62.60 & 41.37 & 35.73 & 47.05 \\
\hline STDEV & & & 11.09 & 55.13 & 14.76 & 13.18 & 11.09 \\
\hline SKEW & & & 0.27 & 0.60 & 0.36 & 0.33 & 0.27 \\
\hline KURT & & & -0.87 & -1.19 & -1.07 & -0.92 & -0.87 \\
\hline \multicolumn{2}{|c|}{ Global Average } & & - & 59.00 & 420.00 & 32.00 & 30.00 \\
\hline
\end{tabular}


Table 2. Summary of the estimated radiological impact parameters (RIP).

\begin{tabular}{|c|c|c|c|c|c|c|c|c|c|c|}
\hline $\begin{array}{l}\text { SAMPLE } \\
\text { Code }\end{array}$ & $D_{\text {in }}\left(n G y h^{-1}\right)$ & D out $\left(n G y h^{-1}\right)$ & $\begin{array}{c}\mathrm{AED}_{\text {outdoor }} \\
\left(\mathrm{mSvy}^{-1}\right)\end{array}$ & $\begin{array}{l}\mathrm{AED}_{\text {indoor }} \\
\left(\mathrm{mSvy}^{-1}\right)\end{array}$ & $\begin{array}{c}\mathrm{Ra}_{\mathrm{eq}} \\
\left(\mathrm{Bqkg}^{-1}\right)\end{array}$ & $\mathrm{H}_{\mathrm{ext}}$ & $\mathrm{H}_{\text {int }}$ & RLI & $\begin{array}{c}\text { ELCR } \\
\left(\mathrm{X} 10^{-3}\right)\end{array}$ & $\begin{array}{c}\text { AGED } \\
\left(\mathrm{mSvy}^{-1}\right)\end{array}$ \\
\hline ISS1 & 84.03 & 44.85 & 0.06 & 0.41 & 102.11 & 0.28 & 0.34 & 0.72 & 1.44 & 0.31 \\
\hline ISS2 & 69.03 & 36.64 & 0.04 & 0.34 & 83.57 & 0.23 & 0.30 & 0.58 & 1.19 & 0.25 \\
\hline ISS3 & 92.46 & 49.07 & 0.06 & 0.45 & 110.77 & 0.30 & 0.38 & 0.78 & 1.59 & 0.34 \\
\hline ISS4 & 92.44 & 49.03 & 0.06 & 0.45 & 111.47 & 0.30 & 0.40 & 0.78 & 1.59 & 0.34 \\
\hline ISS5 & 69.88 & 36.63 & 0.04 & 0.34 & 82.50 & 0.22 & 0.33 & 0.57 & 1.20 & 0.25 \\
\hline ISS6 & 61.39 & 32.62 & 0.04 & 0.30 & 74.00 & 0.20 & 0.26 & 0.52 & 1.05 & 0.23 \\
\hline ISS7 & 126.98 & 66.49 & 0.08 & 0.62 & 148.26 & 0.40 & 0.58 & 1.04 & 2.18 & 0.46 \\
\hline ISS8 & 118.97 & 62.06 & 0.08 & 0.58 & 137.25 & 0.37 & 0.54 & 0.97 & 2.04 & 0.43 \\
\hline ISS9 & 93.85 & 48.52 & 0.06 & 0.46 & 106.17 & 0.29 & 0.45 & 0.75 & 1.61 & 0.33 \\
\hline ISS10 & 74.64 & 39.18 & 0.05 & 0.37 & 87.11 & 0.24 & 0.33 & 0.62 & 1.28 & 0.27 \\
\hline ISS11 & 60.33 & 31.88 & 0.04 & 0.30 & 71.90 & 0.20 & 0.26 & 0.50 & 1.04 & 0.22 \\
\hline ISS12 & 84.05 & 44.73 & 0.05 & 0.41 & 102.37 & 0.28 & 0.36 & 0.71 & 1.44 & 0.31 \\
\hline ISS13 & 98.79 & 52.23 & 0.06 & 0.48 & 118.86 & 0.32 & 0.44 & 0.82 & 1.70 & 0.36 \\
\hline ISS14 & 98.39 & 52.49 & 0.06 & 0.48 & 120.52 & 0.33 & 0.42 & 0.84 & 1.69 & 0.36 \\
\hline ISS15 & 58.68 & 30.68 & 0.04 & 0.29 & 67.58 & 0.18 & 0.26 & 0.48 & 1.01 & 0.21 \\
\hline ISS16 & 59.12 & 30.83 & 0.04 & 0.29 & 69.00 & 0.19 & 0.28 & 0.48 & 1.02 & 0.21 \\
\hline ISS17 & 67.77 & 35.15 & 0.04 & 0.33 & 78.30 & 0.21 & 0.34 & 0.54 & 1.16 & 0.24 \\
\hline ISS18 & 63.99 & 33.02 & 0.04 & 0.31 & 73.14 & 0.20 & 0.32 & 0.50 & 1.10 & 0.22 \\
\hline ISS19 & 113.97 & 60.08 & 0.07 & 0.56 & 135.10 & 0.37 & 0.50 & 0.95 & 1.96 & 0.41 \\
\hline ISS20 & 107.00 & 56.34 & 0.07 & 0.52 & 126.88 & 0.34 & 0.48 & 0.89 & 1.84 & 0.39 \\
\hline ISS21 & 106.17 & 55.65 & 0.07 & 0.52 & 125.55 & 0.34 & 0.50 & 0.86 & 1.82 & 0.38 \\
\hline ISS22 & 63.06 & 33.20 & 0.04 & 0.31 & 75.09 & 0.20 & 0.29 & 0.52 & 1.08 & 0.23 \\
\hline ISS23 & 96.99 & 50.07 & 0.06 & 0.48 & 111.20 & 0.30 & 0.49 & 0.76 & 1.67 & 0.34 \\
\hline ISS24 & 63.43 & 33.62 & 0.04 & 0.31 & 76.53 & 0.21 & 0.28 & 0.53 & 1.09 & 0.23 \\
\hline ISS25 & 135.68 & 71.16 & 0.09 & 0.67 & 160.77 & 0.44 & 0.64 & 1.11 & 2.33 & 0.49 \\
\hline ISS26 & 90.09 & 46.82 & 0.06 & 0.44 & 104.65 & 0.28 & 0.44 & 0.72 & 1.55 & 0.32 \\
\hline ISS27 & 76.41 & 39.47 & 0.05 & 0.37 & 87.59 & 0.24 & 0.39 & 0.60 & 1.31 & 0.27 \\
\hline ISS28 & 77.99 & 40.44 & 0.05 & 0.38 & 88.44 & 0.24 & 0.36 & 0.63 & 1.34 & 0.28 \\
\hline ISS29 & 93.42 & 49.45 & 0.06 & 0.46 & 110.93 & 0.30 & 0.39 & 0.79 & 1.60 & 0.34 \\
\hline ISS30 & 76.90 & 40.38 & 0.05 & 0.38 & 89.45 & 0.24 & 0.33 & 0.64 & 1.32 & 0.28 \\
\hline ISS31 & 125.31 & 66.40 & 0.08 & 0.61 & 150.68 & 0.41 & 0.54 & 1.05 & 2.15 & 0.46 \\
\hline ISS32 & 106.17 & 55.65 & 0.07 & 0.52 & 125.55 & 0.34 & 0.50 & 0.86 & 1.82 & 0.38 \\
\hline ISS33 & 95.39 & 50.56 & 0.06 & 0.47 & 114.46 & 0.31 & 0.41 & 0.80 & 1.64 & 0.35 \\
\hline ISS34 & 69.03 & 36.64 & 0.04 & 0.34 & 83.57 & 0.23 & 0.30 & 0.58 & 1.19 & 0.25 \\
\hline ISS35 & 109.88 & 58.63 & 0.07 & 0.54 & 133.41 & 0.36 & 0.45 & 0.94 & 1.89 & 0.41 \\
\hline ISS36 & 92.44 & 49.03 & 0.06 & 0.45 & 111.47 & 0.30 & 0.40 & 0.78 & 1.59 & 0.34 \\
\hline ISS37 & 69.88 & 36.63 & 0.04 & 0.34 & 82.50 & 0.22 & 0.33 & 0.57 & 1.20 & 0.25 \\
\hline ISS38 & 85.25 & 44.60 & 0.05 & 0.42 & 99.94 & 0.27 & 0.40 & 0.69 & 1.46 & 0.31 \\
\hline ISS39 & 88.82 & 46.07 & 0.06 & 0.44 & 101.08 & 0.27 & 0.41 & 0.71 & 1.53 & 0.32 \\
\hline ISS40 & 114.43 & 59.77 & 0.07 & 0.56 & 132.31 & 0.36 & 0.52 & 0.93 & 1.96 & 0.41 \\
\hline ISS41 & 69.88 & 36.63 & 0.04 & 0.34 & 82.50 & 0.22 & 0.33 & 0.57 & 1.20 & 0.25 \\
\hline ISS42 & 96.61 & 50.31 & 0.06 & 0.47 & 112.29 & 0.30 & 0.46 & 0.78 & 1.66 & 0.34 \\
\hline ISS43 & 99.05 & 51.21 & 0.06 & 0.49 & 112.19 & 0.30 & 0.47 & 0.79 & 1.70 & 0.35 \\
\hline ISS44 & 118.97 & 62.06 & 0.08 & 0.58 & 137.25 & 0.37 & 0.54 & 0.97 & 2.04 & 0.43 \\
\hline ISS45 & 102.94 & 53.08 & 0.07 & 0.50 & 116.05 & 0.31 & 0.50 & 0.81 & 1.77 & 0.36 \\
\hline ISS46 & 74.64 & 39.18 & 0.05 & 0.37 & 87.11 & 0.24 & 0.33 & 0.62 & 1.28 & 0.27 \\
\hline ISS47 & 60.33 & 31.88 & 0.04 & 0.30 & 71.90 & 0.20 & 0.26 & 0.50 & 1.04 & 0.22 \\
\hline
\end{tabular}


Continuation of Table 2. Summary of the estimated radiological impact parameters (RIP).

\begin{tabular}{|c|c|c|c|c|c|c|c|c|c|c|}
\hline $\begin{array}{c}\text { SAMPLE } \\
\text { Code }\end{array}$ & $D_{\text {in }}\left(\mathrm{nGyh}^{-1}\right)$ & $\mathrm{D}_{\text {out }}\left(\mathrm{nGyh}^{-1}\right)$ & $\begin{array}{c}\mathrm{AED}_{\text {outdoor }} \\
\left(\mathrm{mSvy}^{-1}\right)\end{array}$ & $\begin{array}{c}\mathrm{AED}_{\text {indoor }} \\
\left(\mathrm{mSvy}^{-1}\right)\end{array}$ & $\begin{array}{c}\mathrm{Ra}_{\text {eq }} \\
\left(\mathrm{Bqkg}^{-1}\right)\end{array}$ & $\mathrm{H}_{\text {ext }}$ & $\mathrm{H}_{\text {int }}$ & RLI & $\begin{array}{c}\text { ELCR } \\
\left(\mathrm{X} \mathrm{10}^{-3}\right)\end{array}$ & $\begin{array}{c}\text { AGED } \\
\left(\mathrm{mSvy}^{-1}\right)\end{array}$ \\
\hline ISS48 & 84.05 & 44.73 & 0.05 & 0.41 & 102.37 & 0.28 & 0.36 & 0.71 & 1.44 & 0.31 \\
\hline ISS49 & 113.97 & 60.57 & 0.07 & 0.56 & 138.60 & 0.38 & 0.50 & 0.96 & 1.96 & 0.42 \\
\hline ISS50 & 125.00 & 65.77 & 0.08 & 0.61 & 149.07 & 0.40 & 0.58 & 1.03 & 2.15 & 0.45 \\
\hline Min & 58.68 & 30.68 & 0.04 & 0.29 & 67.58 & 0.18 & 0.26 & 0.48 & 1.01 & 0.21 \\
\hline Max & 135.68 & 71.16 & 0.09 & 0.67 & 160.77 & 0.44 & 0.64 & 1.11 & 2.33 & 0.49 \\
\hline Mean & 89.56 & 47.04 & 0.06 & 0.44 & 105.63 & 0.29 & 0.41 & 0.74 & 1.54 & 0.32 \\
\hline Median & $\mathbf{9 1 . 2 7}$ & 47.67 & 0.06 & 0.45 & 105.41 & 0.29 & 0.40 & 0.73 & 1.57 & 0.33 \\
\hline STDEV & $\mathbf{2 0 . 9 1}$ & 11.00 & 0.01 & 0.10 & 24.81 & 0.07 & 0.10 & 0.17 & 0.36 & 0.08 \\
\hline SKEW & 0.27 & 0.28 & 0.28 & 0.27 & 0.29 & 0.29 & 0.32 & 0.27 & 0.27 & 0.27 \\
\hline KURT & -0.87 & -0.88 & -0.88 & -0.87 & -0.85 & -0.85 & -0.76 & -0.91 & -0.87 & -0.91 \\
\hline $\begin{array}{c}\text { Acceptable } \\
\text { LIMIT }\end{array}$ & 84.00 & 59.00 & 0.07 & 0.41 & 370.00 & $\leq 1$ & $\leq 1$ & $\leq 1$ & 3.75 & 0.30 \\
\hline
\end{tabular}

\section{DISCUSSION}

A well calibrated Super-Spec (RS-125) gamma spectrometer was used to measure the activity concentrations of ${ }^{40} \mathrm{~K},{ }^{238} \mathrm{U},{ }^{232} \mathrm{Th}$ and gamma doses rate at $1 \mathrm{~m}$ above the ground level over a laterite mining field in Ilorin, Kwara State, North-central Nigeria. Measurements were carried out in 50 randomly selected sample points. The overall mean activity concentrations of ${ }^{40} \mathrm{~K},{ }^{238} \mathrm{U},{ }^{232} \mathrm{Th}$ and gamma dose are ${ }^{2} 5.7^{2}$, 43.89, $38.79 \mathrm{Bqkg}^{-1}$, and $46.44 \mathrm{nGyh}^{-1}$ respectively. To further study the distribution of these measured radionuclides and the gamma dose rate, isopotassium, isouranium, isothorium and isodose maps of the laterite mining field were plotted using Surfer 15 software and presented in figures 2 to 5 respectively. Two colors (green and red) were used to project areas with values greater than the recommended limits provided by UNSCEAR. Green field represents areas within the recommended limits while red which is universally used for caution/danger, represent areas whose values are above the recommended average. The iso-maps revealed that the mine field is blessed with uranium and thorium which in turn contributes to the dose rate. The enhancement of the dose rate caused by these radionuclides is evident in figure 5 as some red fields can be seen.

Correlation analysis was further conducted to study the relationship between these measured radionuclides and the gamma dose rate and the result are presented in table 3 . The results were classified according to the correlation coefficient $\mathrm{R}{ }^{(49)}$, as follows:

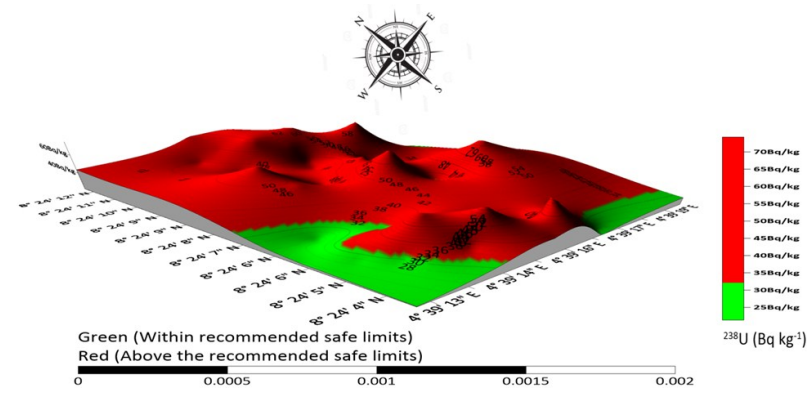

Figure 3. Isouranium map of the laterite mining field.

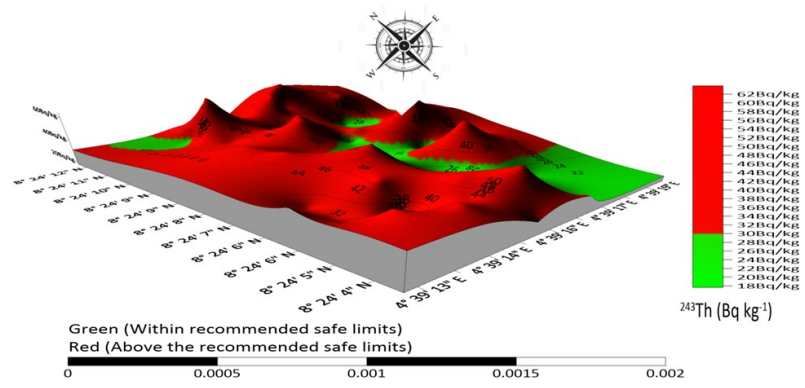

Figure 4. Isothorium map of the laterite mining field.

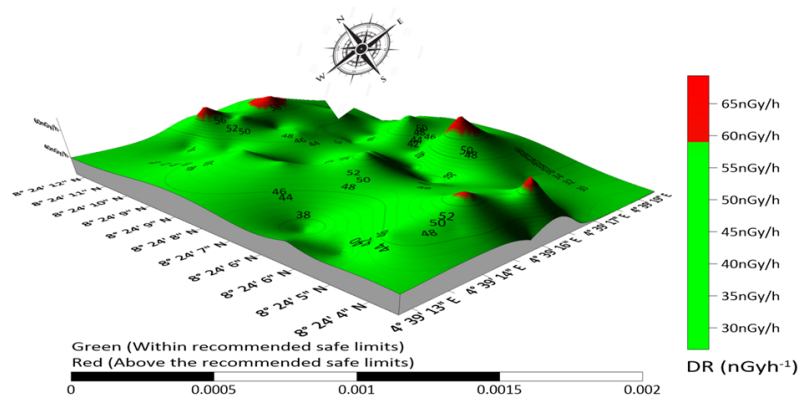

Figure 5. IsoDose-rate map of the laterite mining field.

Int. J. Radiat. Res., Vol. 18 No. 4, October 2020 
Table 3. Pearson's correlation matrix showing the relationship between the measured radionuclides and dose rate.

\begin{tabular}{|c|c|c|c|c|}
\hline & $\begin{array}{c}D R \\
\left(n G y h^{-1}\right)\end{array}$ & $\begin{array}{c}{ }^{40} K \\
\left(B q k g^{-1}\right)\end{array}$ & $\begin{array}{c}{ }^{238} U \\
\left(B q k g^{-1}\right)\end{array}$ & $\begin{array}{c}{ }^{232} \mathrm{Th}^{-} \\
\left(\mathrm{Bqkg}^{-1}\right)\end{array}$ \\
\hline$D R\left(n G y h^{-1}\right)$ & 1.0000 & & & \\
\hline${ }^{40} K\left(B q k g^{-1}\right)$ & 0.3130 & 1.0000 & & \\
\hline${ }^{238} U\left(B q k g^{-1}\right)$ & 0.6633 & 0.1975 & 1.0000 & \\
\hline${ }^{232} \mathrm{Th}\left(\mathrm{Bqkg}{ }^{-1}\right)$ & 0.7225 & 0.0000 & 0.0071 & 1.0000 \\
\hline
\end{tabular}

$0.8 \leq|\mathrm{R}| \leq 1$ suggests a strong correlation; $0.5 \leq|\mathrm{R}| \leq 0.8$ suggests a significant correlation; $0.3 \leq|\mathrm{R}| \leq 0.5$ suggests a weak correlation; and $|\mathrm{R}|<0.3$ suggests an insignificant correlation.

A somewhat weak correlation of 0.3130 exists between gamma dose rate $(D R)$ and ${ }^{40} \mathrm{~K}$, a moderately significant correlation of 0.6633 exists between $D R$ and ${ }^{238} \mathrm{U}$ and a significant correlation of 0.7225 as found to exist between $D R$ and ${ }^{232} \mathrm{Th}$. The correlation results showed that the mine field is loaded with uranium and thorium, with ${ }^{232} \mathrm{Th}$ contributing more signi significant to the gamma dose received from the field than ${ }^{238} \mathrm{U}$ and ${ }^{40} \mathrm{~K}$. These radionuclides have been noted for their notorieties and contributions to background ionizing radiation which is linked with various kinds of cancers, liver diseases and ruthless health related harms which could eventually lead to death $(16,17,19,50$, 51,52 ).

In general, comparative analysis of these mean values of ${ }^{40} \mathrm{~K},{ }^{238} \mathrm{U},{ }^{232} \mathrm{Th}$ and $D R$ for the Laterite mine field under study with some selected studies from literatures across the world is given in table 4 . It was observed that the mean values of $238 \mathrm{U}$ obtained in this study is only higher than the values obtained by [41] in India, [42] in Turkey for Clay soil, [48] in Ghana and the findings of [19] and [14] from Nigeria. The values of ${ }^{40} \mathrm{~K}$ even though lower than recommended limit of $420 \mathrm{Bg} \mathrm{kg}^{-1}$, it compares well with the values reported by most of the authors (see table 4). The mean values of ${ }^{238} \mathrm{U}$ obtained in this study compares relatively with works reported by most authors except for (44) and few others whose values are much higher.
The variation observed in the activity concentrations of these radionuclides when compared with other studies was believed to be because these radio-elements are not evenly spread in the earth crust. So their concentration level depends mostly on the local geology.

The results of the activity concentrations of these radionuclides were used to estimate the corresponding radiation hazard parameters to assess the suitability of the granite for building and construction purposes. The estimated radium equivalent $\left(R a_{e q}\right)$ ranges between 160.77 and $67.58 \mathrm{~Bq} \mathrm{~kg}^{-1}$ with average value of 105.63 $B q \mathrm{~kg}^{-1}$. These estimated values of $R a_{e q}$ are below the limit of $370 \mathrm{~Bq} \mathrm{~kg}^{-1}$ (31) for the use of materials in the construction of buildings. The external radiation hazards $\left(H_{e x t}\right)$ and the internal radiation hazard $\left(H_{\text {int }}\right)$ calculated were below unity as recommended by UNSCEAR. The representative level index $(R L I)$, a radiological hazard parameter used as screening tool for identifying building materials that may possibly be of radiological concern to be used as construction materials. The estimated RLI ranged from 0.48 to 1.11 with an average value of 0.78 . About $12 \%$ of the estimated values are greater than the recommended value of 1 . Even though the mean value is less than unity but the fact that some values are higher than 1 , care should be taken in the use of the lateritic soil from this mine field for building and construction. The estimated values for the ELCR were below the recommended limits of $3.75 \times 10$ -3. While estimated mean values of $A G E D$ for the residents using the laterite for building was evaluated and found to be higher than the recommended limit of $0.3 \mathrm{mSvy}^{-1}$ (i.e 0.32 $\left.m S v y^{-1}\right)$. The high values of AGED further amplified our concerns in the use of the lateritic soil from this mine field for building and construction purposes. The contributions of ${ }^{40} \mathrm{~K}$, ${ }^{238} U$ and ${ }^{232} \mathrm{Th}$ to the hazard parameters $\left(D_{\text {out }} D_{\text {in, }}\right.$ $R a_{e q}, H_{i n}, H_{e x t}, R L I$ and AGED) are presented in figure 6 and 7. It's evident from these plots that ${ }^{234} \mathrm{Th}$ is the principal contributor to the radiation hazards. 
Table 4. Comparison of the mean activity concentration and dose rate with some selected studies.

\begin{tabular}{|c|c|c|c|c|c|c|}
\hline Case Study & $U-238\left(B q k^{-1}\right)$ & Th-232 (Bq kg-1) & $K-40\left(B q k^{-1}\right)$ & Dose rate $\left(n G y h^{-1}\right)$ & Country & References \\
\hline Soil & 19.16 & 48.56 & 1146.88 & 89.60 & India & (41) \\
\hline Kaolin (soil) & 82.00 & 94.80 & 463.60 & 117.70 & Turkey & (42) \\
\hline Clay (soil) & 39.30 & 49.60 & 569.50 & 74.10 & Turkey & (42) \\
\hline Floor ceramic & 101.22 & 87.53 & 304.57 & 213.98 & Iraq & (43) \\
\hline Wall ceramic & 102.12 & 70.90 & 328.60 & 178.40 & Iraq & (43) \\
\hline Kaolin (soil) & 964.70 & 251.60 & 58.90 & 58.10 & Eqypt & (44) \\
\hline Phosphogypsum & 206.80 & 99.10 & 15.10 & 154.60 & Brazil & (45) \\
\hline Kaolin (soil) & 38.20 & 65.10 & 93.90 & 59.60 & $\begin{array}{c}\text { Nigeria } \\
\text { (Ifonyintedo) }\end{array}$ & (19) \\
\hline Building materials & 51.50 & 48.10 & 114.70 & - & Australia & (46) \\
\hline Sands (soil) & 78.00 & 33.00 & 337.00 & - & Egypt & (47) \\
\hline Soil Samples & 55.30 & 26.40 & 505.10 & 66.30 & $\begin{array}{c}\text { Nigeria } \\
\text { (Itagunmodi) }\end{array}$ & (15) \\
\hline Soil and Rock & 13.60 & 24.20 & 162.10 & - & Ghana & (48) \\
\hline Laterite (soil) & 30.00 & 41.00 & 65.00 & 17.510 & $\begin{array}{c}\text { Nigeria } \\
\text { (Obajana) }\end{array}$ & (14) \\
\hline Laterites (Soil) & 43.89 & 38.79 & 81.38 & 46.44 & $\begin{array}{l}\text { Nigeria } \\
\text { (Ilorin) }\end{array}$ & Present Study \\
\hline Soil and Rock & 32.00 & 30.00 & 420.00 & 59.00 & Global Limit & (31) \\
\hline
\end{tabular}

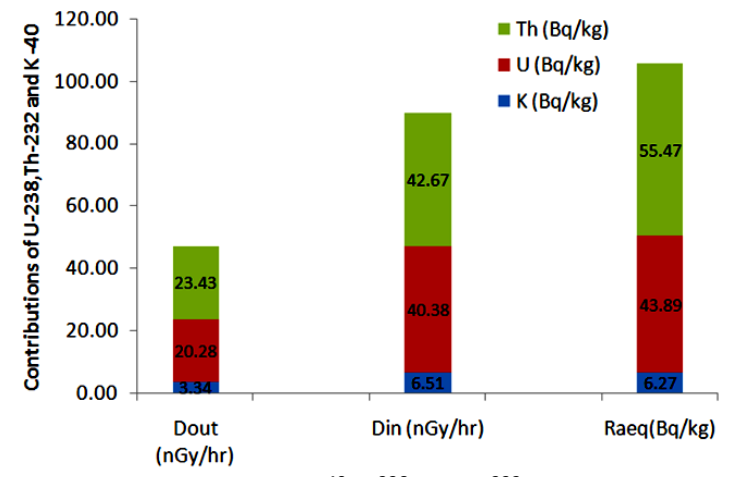

Figure 6. Contributions of ${ }^{40} \mathrm{~K},{ }^{238} \mathrm{U}$ and ${ }^{232} \mathrm{Th}$ to $\mathrm{D}_{\text {out }}, \mathrm{D}_{\text {in }}$ and $\mathrm{Ra}_{\mathrm{eq}}$.

\section{CONCLUSIONS}

The measured radionuclides and the estimated hazard indices are mostly higher than the recommended limits, which implies that the risk of radiation exposure is high for this lateritic soil. The research hereby recommends that radionuclide monitoring and assessment of all mined minerals used as building materials in Kwara State and the country at large carried out on a regular basis, and appropriate authority should implement specific statutory requirements and laws to regulate the high rate of mining activities. Also, in accordance with international recommendations quoted in the Basic Safety Series No.115 from the IAEA, the use of building materials containing enhanced

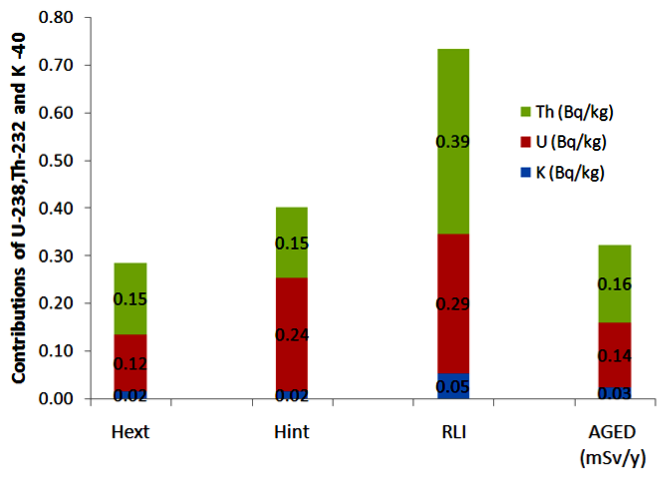

Figure 7. Contributions of ${ }^{40} \mathrm{~K},{ }^{238} \mathrm{U}$ and ${ }^{232} \mathrm{Th}$ to $\mathrm{H}_{\mathrm{ext}}, \mathrm{H}_{\mathrm{in}}, \mathrm{RLI}$ and AGED.

concentrations of NORM should be controlled and restricted under the application of the radiation safety standards.

\section{ACKNOWLEDGEMENT}

This research was granted by the University of Ilorin Staff Development Award (SDA) for PhD program and partial funding was received via the University of Ilorin Institutional Based Research TED Fund program (IBR TED Fund 12 batch RP Disbursement). We also acknowledge the support received from Covenant University.

Int. J. Radiat. Res., Vol. 18 No. 4, October 2020 
Conflicts of interest: Declared none.

\section{REFERENCES}

1. Abdus Saleque K (2008) Social and Environmental Impacts of Mining-Australian Lessons on Mitigation.Website:http://

phulbariproject.wordpress.com/2008/10/28/\%E2\%80\% 9Csocialand-environmental-impacts-of-mining-australianlessons-on-mitigation\%E2\%80\%9D/. (Accessed 16 April 2019).

2. Orosun MM, Tchokossa P, Lawal TO, Bello SO, Ige SO and Nwankwo LI (2016) Assessment of heavy metal pollution in drinking water due to mining and smelting activities in Ajaokuta. Nigerian Journal of Technological Development, 13: $30-38$.

3. Oluyide SO, Tchokossa P, Orosun MM, Akinyose FC, Louis $H$, Ige SO (2018) Natural Radioactivity and Radiological Impact Assessment of Soil, Food and Water around Iron and Steel Smelting Area in Fashina Village, Ile-Ife, Osun State, Nigeria. Journal of Applied Sciences and Environmental Management, 23 (1): 135-143.

4. Orosun MM, Usikalu RM, Oyewumi KJ, Adagunodo AT, (2019) Natural Radionuclides and Radiological Risk Assessment of Granite Mining Field in Asa, North-central Nigeria. MethodsX. 6: 2504-2514. doi:https://doi.org/10.1016/ j.mex.2019.10.032

5. Jibiri NN and Esen NU (2011) Radionuclide contents and radiological risk to the population due to raw minerals and soil samples from the mining sites of quality ceramic and pottery industries in Akwa lbom, Nigeria. Radioprotection, 46(1): $75-87$.

6. Usikalu MR, Anoka OC, Balogun FA (2011) Radioactivity measurement of Jos Tin mine tailings. Achives of Physics Research, 2(2): 80-86.

7. Oyelami CA and Van Rooy JL (2016) A review of the use of lateritic soils in the construction/development of sustainable housing in Africa: A geological perspective. Journal of African Earth Sciences, 119: 226-237.

8. Usikalu MR, Fuwape IA, Jatto SS, Awe OF, Rabiu AB, Achuka JA (2017) Assessment of radiological parameters of soil in Kogi State, Nigeria, Environmental Forensics, 18(1): 1-14. 9. Orosun. MM, Lawal TO, Ezike SC, Salawu NB, Atolagbe BM, Akinyose FC, Ige SO and Martins G (2017) Natural radionuclide concentration and radiological impact assessment of soil and water from Dadinkowa Dam, Northeast Nigeria. Journal of the Nigerian Association of Mathematical Physics, 42 (1): $307-316$.

10. Farai IP and Ademola JA (2001) Population dose due to building materials in Ibadan, Nigeria. Radiat Prot Dosim, 95: 69-73.

11. Ademola JA (2005) Radionuclide content of concrete building blocks and radiation dose rates in some dwellings in Ibadan, Nigeria. J Environ Radioact, 81: 107-113.

12. Obed RI, Farai IP, Jibiri NN (2005) Population dose distribution due to soil radioactivity concentration levels in 18 cities across Nigeria. J Radiol Prot, 25: 305-312.

13. Ademola AK, Hammed OS, \& Adejumobi CA (2008) Radioactivity and dose assessment of marble samples from Igbeti mines, Nigeria. Radiation Protection Dosimetry, 132 (1): 94-97.

14. Ajayi JO, Balogun BB and Olabisi O (2012) Natural Radionuclide Contents in Raw Materials and the Aggregate Fin- ished Product from Dangote Cement PIc, Obajana, Kogi State, and North Central Nigeria. Res J Environ Earth Sci, 4 (11): 959-961.

15. Ademola AK, Bello AK, Adejumobi AC (2014) Determination of natural radioactivity and hazard in soil samples in and around gold mining area in Itagunmodi, southwestern, Nigeria. J Radiat Res App/ Sci, 7(3): 249-255.

16. Isinkaye OM, Jibiri NN, Olomide AA (2015) Radiological health assessment of natural radioactivity in the vicinity of Obajana cement factory, North Central Nigeria. J Med Phys, 40(1): 52-59.

17. Orosun MM, Lawal TO, Akinyose FC (2016) Natural radionuclide concentrations and radiological impact assessment of soil and water in Tanke-llorin, Nigeria. Zimbabwe Journal of Science \& Technology, 11: 158-172.

18. Omeje M, Adewoyin OO, Joel ES, Ehi-Eromosele CO, Emenike CP, Usikalu MR, Akinwumi SA, Zaidi E and Mohammad AS (2018) Natural radioactivity concentrations of 226Ra, 232Th, and 40K in commercial building materials and their lifetime cancer risk assessment in Dwellers, Human and Ecological Risk Assessment: An International Journal, 24(8): 2036-2053.

19. Adagunodo TA, Hammed OS, Usikalu MR, Ayara WA Ravisankar R (2018) Data sets on the radiometric Survey over a Kaolinitic Terrain in Dahomey Basin, Nigeria. Data in Brief, 18: 814-822.

20. Anifowoshe AT, Owolodun OA, Oyinlola BO, Abdulganiyu K, Yusuf RD, Oredein OA, Iyiola OA (2018) Incidence of Common and Rare Cancers in Ilorin, Nigeria. Notulae Scientia Biologicae, 10(4): 453-459.

21. Oyegun RO (1985) The use and waste of water in a third world city. GeoJournal, 10(2): 205-210.

22. Megwara JU and Udensi EE (2014) Structural analysis using aeromagnetic data: case study of parts of Southern Bida Basin, Nigeria and the surrounding basement rocks. Earth Science Research, 3(2).

23. Kayode JS, Nawawi MNM, Baioumy HM, Khalil AE, \& Khiruddin BA (2015) Delineation of the subsurface geological structures of Omu-Aran area, south-western Nigeria, using aeromagnetic data.

24. Orosun MM, Oniku AS, Adie P, Orosun OR, Salawu NB, and Louis H (2020) Magnetic susceptibility measurement and heavy metal pollution at an automobile station in llorin, North-Central Nigeria, Environmental Research Communication 2(2020): 015001. https://doi.org/10.1088/2515$7620 / a b 636 a$

25. Usikalu MR, Onumejor CA, Akinpelu A, Achuka JA, Omeje $\mathrm{M}$ and Oladapo OF (2018) Natural radioactivity concentration and its health implication on dwellers in selected locations of Ota, IOP Conf. Series: Earth and Environmental Science, 173.

26. International Atomic Energy Agency (IAEA) (1989) Construction and use of calibration facilities for radiometric field equipment. Technical reports series no. 309, IAEA, Vienna.

27. Omeje M, Wagiran H, Ibrahim N, Lee SK, Sebri S (2014) Radiological monitoring of borehole in Dei-Dei, Abuja, North Central Nigeria. APCBEE Procedia, 10(2014): 54-58.

28. Oyeyemi KD, Aizebeokhai AP, Olofinnade OM (2017). Dataset on ground radiometric survey in part of the Eastern Dahomey Basin, SW Nigeria. Data in Brief, 15: 148-154.

29. Oyeyemi KD, Usikalu MR, Aizebeokhai AP, Achuka JA, and Jonathan $O$ (2017) Measurements of radioactivity levels in part of Ota Southwestern Nigeria: Implications for radiological hazards indices and excess lifetime cancer-risks. Journal of Physics: Conference Series, 852, 012042. 
30. Ramasamy V, Suresh G, Meenakshisundaram V, Ponnusamy V (2011) Horizontal and vertical characterization of radionuclides and minerals in river sediments. Applied Radiation and Isotopes, 69: 184 - 195.

31. UNSCEAR (2000) Sources, effects and risks of ionization radiation, United Nations Scientific Committee on the Effects of Atomic Radiation. Report to The General Assembly, with Scientific Annexes B: Exposures from Natural Radiation Sources; New York.

32. Akinyose FC, Tchokossa P, Orosun MM, Oluyde SO, Umakha M, Ochommadu KK, Olaniyan TA, Ajibade OA (2018) Radiological Impacts of Natural Radioactivity in Locally Produced Tobacco Products in Ibadan, Oyo State, Nigeria. Momona Ethiopian Journal of Science (MEJS), 10(1): 59-75.

33. Akinyose FC, Tchokossa P, Orosun MM, Oluyide SO, Olatunji O and Martins G (2018) A Study of Natural Radioactivity and Gamma Radiation Hazard in Tobacco Leaves and Cigarettes in Oyo State. Nigeria Manila Journal of Science, 11 (2018): 104-114.

34. European Commission (1999) Radiological protection principles concerning the natural radioactivity of building materials. Radiation Protection 112, Directorate. General, Environment, Nuclear Safety and Civil Protection, European Commission, Luxembourg.

35. Farai IP and Isinkaye MO (2010) Radiological safety assessment of surface-water dam sediments used as building materials in southwestern Nigeria. J Radiol Prot, 29: 8593.

36. Stojanovska Z, Nedelkovski D, Ristova M (2010) Natural radioactivity and human exposure by raw materials and end product from cement industry used in building materials. Radiat Meas, 45: 969-72.

37. Baykara O, Karatepe S, Dogru M (2011) Assessment of natural radioactivity and radiological hazards in construcution materials used in Elazig, Turkey. Radiat Meas, 46: 153 $-158$.

38. Orosun MM, Alabi AB, Olawepo AO, Orosun RO, Lawal TO and Ige SO (2018) Radiological safety of water from Hadejia river. IOP Conf. Series: Earth and Environmental Science 173 (2018).

39. Adagunodo $T$, Sunmonu L, Adabanija M, Omeje M, Odetunmibi O, ljeh V (2019) Statistical assessment of radiation exposure risks of farmers in Odo Oba, Southwestern Nigeria. Bulletin of the Mineral Research and Exploration, 159 (159): 201-217.

40. Normality Testing - Skewness and Kurtosis (2019) Normality Testing - Skewness and Kurtosis. Available at: https:// help.gooddata.com/doc/en/reporting-and-dashboards/ maql-analytical-query-language/maql-expressionreference/aggregation-functions/statistical-functions/ predictive-statistical-use-cases/normality-testingskewness-and-kurtosis (Accessed on 15th July, 2019).

41. Chandrasekaran A, Ravisankar R, Senthilkumar G, Thillaivelavan K, Dhinakaran B, Vijayagopal P, Bramha SN, Venkatraman B (2014) Spatial distribution and life time cancer risk due to gamma radioactivity in Yelagiri Hills,Tamilnadu, India, Egypt. J Basic App/ Sci, 1: 38-48.

42. Turhan, S (2009) Radiological impacts of the usability of clay and kaolin as raw material in manufacturing of structural building materials in Turkey. Journal of Radiological Protection, 29(1): 75-83.

43. Amana MS (2017) Radiation hazard index of common imported ceramic using for building materials in Iraq, Aust. $J$ Basic App/ Sci, 11(10): 94-102.

44. El-Dine NW, Sroor A, El-Shershaby A, El-Bahi SM, Ahmed F (2004) Radioactivity in local and imported kaolin types used in Egypt. Appl Radiat Isot, 60: 105-109.

45. Mazzilli B and Saueia C (1999) Radiological implications of using phosphogypsum as a building material in Brazil. Radiat Prot Dosim, 86(1): 63-67.

46. Berekta J and Mathew PJ (195) Natural radioactivity in Australian building materials, industrial waste and byproduct. Health Physics, 48: 87-95.

47. El-Afifi EM, Hilal MA, Khalifa SM, Aly HF (2006) Evaluation of $U, T h, K$ and emanated radon in some NORM and TENORM samples. Radiation Measurements, 41: 627-633.

48. Faanu A, Darko EO, Ephraim JH (2011) Determination of natural radioactivity and hazard in soil and rock samples in a mining area in Ghana. West African Journal of Applied Ecology, 19: 77-92.

49. Yu J and Hu X (2005). Application of data statistical analysis with SPSS. Post \& Telecom Press, Beijing, 1: 163-173.

50. Akinnagbe DM, Orosun MM, Orosun RO, Osanyinlusi $O$, Yusuk KA, Akinyose FC, Olaniyan TA, Ige SO (2018) Assessment of Radon Concentration of Ground Water in ljero Ekiti. Manila Journal of Science, 11: 32-41.

51. Oluyide SO, Tchokossa P, Akinyose FC, Orosun MM (2018) Assessment of radioactivity levels and transfer factor of natural radionuclides around iron and steel smelting company located in Fashina village, lle-ife, Osun state, Nigeria. Facta Universitatis, Series. Working and Living Environmental Protection, 15(3): 241-256.

52. Adagunodo TA, George Al, Ojoawo IA, Ojesanmi K, \& Ravisankar R (2018) Radioactivity and radiological hazards from a kaolin mining field in Ifonyintedo, Nigeria. MethodsX, 5: 362-374. 